\title{
Spectroscopic measurement of the plasma electron density and outflow velocity in a polar coronal hole
}

\author{
E. Antonucci ${ }^{1}$, M. A. Dodero ${ }^{2}$, S. Giordano ${ }^{1}$, V. Krishnakumar ${ }^{3}$, and G. Noci $^{4}$ \\ ${ }^{1}$ Istituto Nazionale di Astrofisica (INAF), Osservatorio Astronomico di Torino, Strada Osservatorio 20, 10025 Pino Torinese, \\ Italy \\ 2 University of Torino, via P. Giuria 1, 10125 Torino, Italy \\ 3 National Solar Observatory, 3083, Corona Loop, PO Box 4, Sunspot, NM 88349, USA \\ ${ }^{4}$ Università di Firenze, Dipartimento di Astronomia e Scienza dello Spazio, Largo E. Fermi 5, 50125 Firenze, Italy
}

Received 30 January 2001 / Accepted 16 October 2003

\begin{abstract}
A new spectroscopic method, aimed to derive the plasma electron density and outflow velocity in expanding solar coronal regions, is discussed in this paper. The method is based on the analysis of a pair of coronal lines emitted via collisional and radiative excitation by the same ion, such as the O VI 1032, $1037 \AA$ doublet. The merit of this technique consists in allowing us to derive at the same time electron density and outflow velocity of the coronal plasma from nearby lines detectable with the same instrument, provided the constraint on mass flux conservation along the flow tube connecting solar corona and heliosphere is taken into account. The results obtained from the analysis of the OVI emission imply that the physical conditions of a polar coronal hole plasma, observed during minimum activity, are the following. The electron density decreases from $4 \times 10^{5} \mathrm{~cm}^{-3}$ at $1.7 R_{\odot}$ to $2-4 \times 10^{4} \mathrm{~cm}^{-3}$ at $3.1 R_{\odot}$, whereas the outflow velocity of the oxygen ions is monotonically increasing to reach $350-500 \mathrm{~km} \mathrm{~s}^{-1}$ at $3.1 R_{\odot}$, depending on the assumptions on the degree of anisotropy of the velocity distribution of the ions. These results of the velocity of expansion of the fast wind confirm those obtained with Doppler dimming techniques when assuming the lowest observed density values for the coronal hole plasma. This implies that, for a rarified corona, the outflow velocity of the fast solar wind in polar holes can be traced by the motion of the O VI ions at least up to $2.4 R_{\odot}$. The analysis also shows that the degree of anisotropy of the oxygen ions, due to the acceleration of the ions across the magnetic field in a coronal hole, exhibits a steep increase and that the geometry of the flow tube diverges very rapidly low down in the inner corona/transition region. The observations of the extended corona analysed in this paper are obtained with the Ultraviolet Coronagraph Spectrometer of the SOHO space mission.
\end{abstract}

Key words. Sun: corona - Sun: UV radiation - Sun: solar wind

\section{Introduction}

The primary source of information on electron density in the solar corona has been so far the polarized visible light emission of the K corona, produced by Thompson scattering of the photospheric radiation on the free electrons of the solar atmosphere. In the inner corona, below $2 R_{\odot}$, the visible light emission can be observed either during eclipses or by groundbased coronagraphs. With the advent of space instruments the field of view of the visible light observations has been considerably extended. At present the LASCO-C3 coronagraph on the SOHO spacecraft observes out to $30 R_{\odot}$. Space instruments have also introduced the capability to derive the electron density by spectral analysis of ultraviolet lines out to approximately $1.3 R_{\odot}$ (e.g., Wilhelm et al. 1998a). In Fig. 1, the electron density of the coronal hole plasma derived from data obtained with visible light coronagraphs operating from space

Send offprint requests to: E. Antonucci,

e-mail: antonucci@to.astro.it
(Guhathakurta et al. 1999; Fisher \& Guhathakurta 1995; Kohl et al. 1998; Munro \& Jackson 1977) are compared with eclipse measurements (e.g., Koutchmy 1977), and with the density obtained from the ratio of the Si VIII 1445 and $1440 \AA$ ultraviolet emission lines, observed in the inner corona by Wilhelm et al. (1998a), with the SUMER spectrometer on SOHO. The density values above $2 R_{\odot}$ can vary by a factor $2-3$, as shown in the figure. It is not straightforward to assess how much the deviation in density values is due to intrinsic variability of solar origin or it is to be ascribed to the use of different instrumentation and diagnostic methods in the detection and analysis of the coronal emission. A variation in density of the order of that given in Fig. 1 at $2 R_{\odot}$ is sufficient to significantly influence the measurement of the outflow velocity in coronal holes (Antonucci et al. 2000, Paper I). Hence we propose an alternative approach that allows us to derive at the same time electron density and outflow velocity in the extended corona. The proposed technique is based on the spectral analysis of intense ultraviolet lines emitted by collisional and radiative excitation, such as 


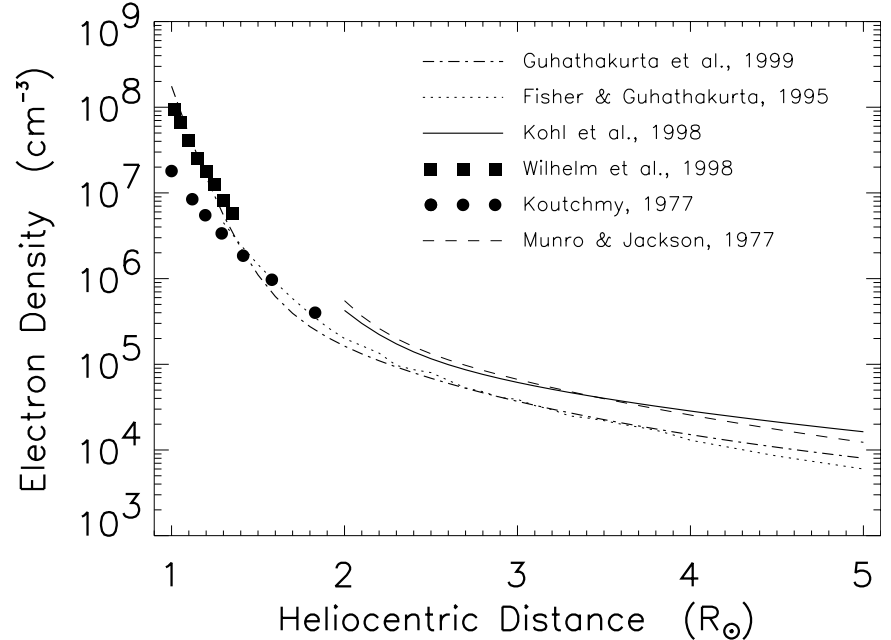

Fig. 1. Coronal electron density as a function of heliocentric distance, obtained with visible light coronagraph data (Koutchmy 1977; Munro \& Jackson 1977; Fisher \& Guhathakurta 1995; Kohl et al. 1998; Guhathakurta et al. 1999) and with ultraviolet spectroscopic observations (Wilhelm et al. 1998a).

the O VI 1032 and $1037 \AA$ lines that can be observed in the extended corona out to a few solar radii with the Ultraviolet Coronagraph Spectrometer (UVCS) on SOHO.

\section{Electron density and outflow velocity diagnostics}

The spectroscopic technique here proposed allows us to derive the electron density and outflow velocity in the general case of an expanding solar corona by using two lines emitted by the same ion, or atom. The method can be applied when the two lines are consisting of both a collisional and a radiative component, or at least one of the two lines has a non-negligible collisional component. This condition is verified for the brightest lines, such as the O VI 1032, 1037 doublet and the HI Ly $\alpha 1216$ and Ly $\beta 1026$ lines, emitted in the extended corona, where resonant scattering becomes important relative to collisional excitation due to the rapid decrease in density with increasing heliocentric distance.

\subsection{Collisional line intensity component}

The intensity of a coronal line excited from the ground level consists in general of both a collisional and a radiative component. The collisional component, expressed in photons $\mathrm{cm}^{-2} \mathrm{~s}^{-1} \mathrm{sr}^{-1}$, has the expression:

$I_{\mathrm{c}}=\frac{1}{4 \pi} b \int_{\text {l.o.s. }} n_{\mathrm{e}} n_{\mathrm{i}} q_{\text {coll }} \mathrm{d} l$,

where $n_{\mathrm{e}}$ is the electron density, $n_{\mathrm{i}}$ is the number density of the ions, or atoms, at the ground level, $b$ is the branching ratio for radiative de-excitation and $q_{\text {coll }}$ is the collisional excitation rate coefficient of the atomic transition (Elwert 1952; Gabriel \& Jordan 1972). The integral is performed along the line-of-sight (l.o.s.). The collisional coefficient is a function of the electron temperature, $T_{\mathrm{e}}$,

$q_{\text {coll }}=2.73 \times 10^{-15} f_{12} \bar{g} \frac{\exp ^{-E_{12} /\left(k_{\mathrm{B}} T_{\mathrm{e}}\right)}}{\sqrt{T_{\mathrm{e}}} E_{12}}$.

The quantity $E_{12}=h v_{0}=h c / \lambda_{0}$ is the energy of the transition, $v_{0}=c / \lambda_{0}$ is the reference frequency and $\lambda_{0}$ is the reference wavelength of the atomic transition, $\bar{g}$ is an effective Gaunt factor, $f_{12}$ is the oscillator strength.

\subsection{Radiative line intensity component}

The radiative component of line intensity (e.g. Noci et al. 1987, 1997 ) is given by the relation

$I_{\mathrm{r}}=\frac{1}{4 \pi} b h v_{0} B_{12} \int_{\text {l.o.s. }} \int_{\Omega} p(\varphi) \mathrm{d} \omega F\left(w_{n}\right) n_{i} \mathrm{~d} l$,

with

$F\left(w_{n}\right)=\int_{-\infty}^{+\infty} I_{\mathrm{ex}}\left[\left(v_{0}+\delta v\left(v_{n}\right)\right), \boldsymbol{n}\right] f_{n}\left(v_{n}-w_{n}\right) \mathrm{d} v_{n}$,

where $I_{\mathrm{ex}}\left(v_{0}+\delta v, \boldsymbol{n}\right)$ is the intensity of the exciting spectrum, emitted from the lower atmosphere along the direction of the incident radiation, $\boldsymbol{n}$. The frequency $\left(v_{0}+\delta v\right)$ at which the absorption occurs depends on the velocity, $v_{n}$, of the absorbing ions along the direction of the incident radiation and the quantity $\delta v=v_{0}(\boldsymbol{v} \cdot \boldsymbol{n}) / c$ is the Doppler shift of the exciting radiation in the frame of reference of the scattering ion. The distribution function of the velocity of the absorbing ions along the direction $\boldsymbol{n}, f\left(v_{n}-w_{n}\right)$, is assumed to be Maxwellian. The vector $\boldsymbol{w}$ represents the mean velocity of the coronal ions in the point of scattering, that, in our case, corresponds to the velocity of expansion of the solar corona. The projection of this vector along the direction of the incident radiation is given by $w_{n}=\boldsymbol{w} \cdot \boldsymbol{n}$. The function $p(\varphi)$ takes into account the geometry of the scattering process, $\varphi$ is the angle between $\boldsymbol{n}$ and the l.o.s., $\mathrm{d} \omega$ is the infinitesimal solid angle around $\boldsymbol{n}$, and $\Omega$ is the solid angle subtended by the solar disk at the point of scattering. $B_{12}$ is the Einstein coefficient for absorption

$B_{12}=\frac{4 \pi^{2} e^{2}}{h v_{0} m_{\mathrm{e}} c} f_{12}$

where $e$ is the charge and $m_{\mathrm{e}}$ is the mass of the electron.

In the formulae (3) and (4), the integral of the product of the intensity of the exciting spectrum and the velocity distribution function of the absorbing ions can be transformed into an integral of the product of the intensity, $I_{\mathrm{ex}}(\lambda)$, and the normalized coronal absorption profile, $\Psi(\lambda, \boldsymbol{n})$, along the direction of the incident radiation, expressed as a function of wavelength $\lambda$

$I_{\mathrm{r}}=\frac{1}{4 \pi} b h \lambda_{0} B_{12} \int_{\text {l.o.s. }} \int_{\Omega} p(\varphi) \mathrm{d} \omega \Phi(\delta \lambda) n_{i} \mathrm{~d} l$,

with

$\Phi(\delta \lambda)=\int_{0}^{+\infty} I_{\mathrm{ex}}(\lambda-\delta \lambda, \boldsymbol{n}) \Psi\left(\lambda-\lambda_{0}\right) \mathrm{d} \lambda$, 
where $\delta \lambda$ is the shift of the disk spectrum introduced by the expansion velocity, $\boldsymbol{w}$, of the coronal absorbing ions/atoms along the direction $n$

$\delta \lambda=\frac{\lambda_{0}}{c} \boldsymbol{w} \cdot \boldsymbol{n}$.

The normalized coronal absorption profile is assumed to be a Gaussian function with standard deviation $\sigma_{\lambda, n}$. This quantity is related to the kinetic temperature of the coronal ions $T_{\mathrm{k}, n}$ along the direction of the incident radiation

$\sigma_{\lambda, n}=\frac{\lambda_{0}}{c} \sqrt{\frac{k_{\mathrm{B}} T_{\mathrm{k}, n}}{m_{\mathrm{p}} A}}$,

where $c$ is the speed of light, $k_{\mathrm{B}}$ is the Boltzmann constant, $m_{\mathrm{p}}$ is the proton mass, $A$ is the ion mass number.

The relation between the integrals $F\left(w_{n}\right)$ and $\Phi(\delta \lambda)$ is the following

$F\left(w_{n}\right)=\frac{\lambda^{2}}{c} \Phi(\delta \lambda)$

\subsection{Method to derive electron density}

The column density of the emitting ions, $\int_{\text {l.o.s. }} n_{i} \mathrm{~d} l$, along the 1.o.s. can be derived from the radiative component of line intensity, given in Eqs. (3) and (6).

$$
\begin{gathered}
\int_{\text {l.o.s. }} n_{i} \mathrm{~d} l \sim \frac{4 \pi I_{\mathrm{r}}}{b h v_{0} B_{12}\left\langle\int_{\Omega} F(w) p(\varphi) \mathrm{d} \omega\right\rangle} \\
=\frac{4 \pi I_{r}}{b h \lambda_{0} B_{12}\left\langle\int_{\Omega} \Phi(\delta \lambda) p(\varphi) \mathrm{d} \omega\right\rangle} .
\end{gathered}
$$

In this approximation the values of the integrals $\left\langle\int_{\Omega} F(\omega) p(\varphi) \mathrm{d} \omega\right\rangle$ and $\left\langle\int_{\Omega} \Phi(\delta \lambda) p(\varphi) \mathrm{d} \omega\right\rangle$ are averages along the 1.o.s.

The same quantity can also be derived by considering the collisional component of a coronal line. Equation (1) can in fact be transformed in:

$\int_{\text {l.o.s. }} n_{i} \mathrm{~d} l \sim \frac{4 \pi I_{\mathrm{c}}}{b\left\langle q_{\text {coll }}\right\rangle\left\langle n_{\mathrm{e}}\right\rangle}$,

where $\left\langle n_{\mathrm{e}}\right\rangle$ and $\left\langle q_{\text {coll }}\right\rangle$ are averages along the 1.o.s.

We can obtain the electron density by equating Eqs. (11) and (12), which is proportional to the ratio of the collisional and radiative components

$\left\langle n_{\mathrm{e}}\right\rangle \sim \frac{I_{\mathrm{c}}}{I_{\mathrm{r}}} \frac{h v_{0} B_{12}}{\left\langle q_{\mathrm{coll}}\right\rangle}\left\langle\int_{\Omega} F(w) p(\varphi) \mathrm{d} \omega\right\rangle$,

and therefore depends on the mean velocity $w$ of the coronal ions.

The values of both the integral containing the Doppler dimming factor, $\left\langle\int_{\Omega} F(w) p(\varphi) \mathrm{d} \omega\right\rangle$, and the electron density, $\left\langle n_{\mathrm{e}}\right\rangle$, are lower than the plane-of-the-sky values by a factor that accounts for the dependence of these quantities along the line of sight. The variation along the l.o.s. of the integral factor is due to that of the solid angle subtended by the solar disk at the point of scattering, combined with the variation of the dimming along the line-of-sight, since both the outflow velocity and the width of the coronal absorbing profile increase with heliodistance. The dependence of electron density along the line-of-sight is related as well to its variation with heliodistance.

In a coronal hole, in the approximation of spherical symmetry, the dimming factor and coronal density are both monotonically decreasing out of the plane of the sky, although the dependence is not the same for the two quantities. The ratio $\rho=\left(\frac{n_{\mathrm{e}} q_{\text {coll }}}{\int_{\Omega} F(w) p(\varphi) \mathrm{d} \omega}\right)_{\text {p.o.s. }} / \frac{\left\langle n_{\mathrm{e}}\right\rangle\left\langle q_{\text {coll }}\right\rangle}{\left\langle\int_{\Omega} F(w) p(\varphi) \mathrm{d} \omega\right\rangle}$, that compares quantities on the plane of the sky (p.o.s.) and averaged along the 1.o.s., has been computed for typical values of O VI ion kinetic temperature and outflow velocity, and of electron density and temperature (we have assumed the parameters used to derive the outflow velocity reported in Fig. 7c, Paper I). This ratio is between 1.2 and 1.5 below $1.7 R_{\odot}$ and close to 1 above $1.7 R_{\odot}$. Thus the ratio $\frac{\left\langle n_{\mathrm{e}}\right\rangle\left\langle q_{\text {coll }}\right\rangle}{\left\langle\int_{\Omega} F(w) p(\varphi) \mathrm{d} \omega\right\rangle}$ can be approximated by its value on the plane of the sky and the Eq. (13) can be rewritten as

$n_{\text {e,p.o.s. }} \approx \frac{I_{\mathrm{c}}}{I_{\mathrm{r}}} \frac{h v_{0} B_{12}}{q_{\text {coll,p.o.s. }}}\left(\int_{\Omega} F(w) p(\varphi) \mathrm{d} \omega\right)_{\text {p.o.s. }}$.

The quantities $\left\langle n_{\mathrm{e}}\right\rangle$ and $n_{\mathrm{e}, \text { p.o.s. }}$ will be simply denoted by $n_{\mathrm{e}}$ in the following sections.

We note that this method does not account for the presence of possible inhomogeneities of quantities such as electron temperature, ion outflow velocity and width of the coronal absorbing profile, since their presence cannot be identified in the observations (in Sect. 3.2.2 the effect of the density inhomogeneities on the results is discussed).

\subsection{Separation of collisional and radiative components of a coronal line}

The diagnostic method discussed above requires the separation of the collisional and radiative contributions to an observed spectral line. The radiative and collisional components, $I_{\mathrm{r}, a}, I_{\mathrm{c}, a}$ and $I_{\mathrm{r}, b}, I_{\mathrm{c}, b}$ of a pair of lines, $a$ and $b$, are related by four equations. The observed line intensities of $a$ and $b$ lines are the sum of the collisional and radiative components:

$I_{\mathrm{r}, a}+I_{\mathrm{c}, a}=I_{\mathrm{obs}, a}$,

$I_{\mathrm{r}, b}+I_{\mathrm{c}, b}=I_{\mathrm{obs}, b}$

The quantities $R_{1}$ and $R_{2}$, are the ratios of the radiative and collisional components of the two lines. Their approximate values are given by the following relations:

$$
\begin{aligned}
\frac{I_{\mathrm{r}, a}}{I_{\mathrm{r}, b}} & =R_{1} \sim \frac{b_{a}}{b_{b}} \frac{B_{12, a}}{B_{12, b}} \frac{\lambda_{0, b}}{\lambda_{0, a}} \frac{\left\langle\int_{\Omega} F_{a}(w) p(\varphi) \mathrm{d} \omega\right\rangle}{\left\langle\int_{\Omega} F_{b}(w) p(\varphi) \mathrm{d} \omega\right\rangle} \\
& =\frac{b_{a}}{b_{b}} \frac{B_{12, a}}{B_{12, b}} \frac{\lambda_{0, a}}{\lambda_{0, b}} \frac{\left\langle\int_{\Omega} \Phi_{a}(\delta \lambda) p(\varphi) \mathrm{d} \omega\right\rangle}{\left\langle\int_{\Omega} \Phi_{b}(\delta \lambda) p(\varphi) \mathrm{d} \omega\right\rangle} \\
\frac{I_{\mathrm{c}, a}}{I_{\mathrm{c}, b}} & =R_{2} \sim \frac{b_{a}}{b_{b}} \frac{\left\langle q_{\mathrm{coll}, a}\right\rangle}{\left\langle q_{\mathrm{coll}, b}\right\rangle}
\end{aligned}
$$


The ratio $R_{1}$ is then a function of the atomic parameters of the transitions, of the exciting spectrum, of the velocity distribution of the coronal absorbing ions along the direction of the incident radiation, that can be expressed in terms of kinetic temperature $T_{\mathrm{k}}$ (Eq. (9)), and of the outflow velocity, $w$, through the functions $F(w)$, or $\Phi(\delta \lambda)$. The ratio $R_{2}$ is a function of the atomic parameters and of the electron temperature, $T_{\mathrm{e}} \cdot R_{1}$ and $R_{2}$ depend on the ratios of quantities averaged along the l.o.s. that can be approximated by their values on the plane of the sky. The radiative and collisional components of the two lines can be then separated by solving the system given by the four Eqs. (15)-(18), accounting for both a static and a non-static corona, whose expansion is considered in the $F(w)$ and $\Phi(\delta \lambda)$ factors.

\section{Analysis}

In our study the coronal hole plasma is analyzed by choosing for diagnostic purposes the ultraviolet lines emitted at $1032 \AA$ and $1037 \AA$ by the O VI ions. These two lines of oxygen 5 times ionized are well-observed even in the dimmest regions of the extended corona. Hence they can be used to determine the electron density in polar coronal holes, sources of the fast solar wind, by adopting the spectroscopic method introduced in Sect. 2. This in fact, as discussed above, accounts for the expansion of the corona in the region of acceleration of the fast wind.

\subsection{Observations}

We apply the density-velocity diagnostics to the observations performed on May 21, 1996, with the Ultraviolet Coronagraph Spectrometer (UVCS). In the time interval from 00.36.31 UT to 10.29.03 UT, the coronagraph scanned the coronal hole present at the North pole in the region between $1.5 R_{\odot}$ and $3.5 R_{\odot}$. The O VI $\lambda 1032$ and 1037 line emissions were detected in an instantaneous field of view (30 arcmin $\times 84$ arcsec) wide, centered above the northern pole. The slit was oriented perpendicular to the radial direction. For each elementary area ( $28 \operatorname{arcsec} \times 84$ arcsec) wide, along the instantaneous field-of-view, the profiles of the O VI lines were detected with spectral resolution $1.1 \AA$. The polar region between $1.5 R_{\odot}$ and $3.5 R_{\odot}$ was scanned in steps increasing with height. In order to improve the statistics, the duration of the observation at any given position was chosen to increase with height up to $2.5 R_{\odot}$ (the exposure time was increased from $1000 \mathrm{~s}$ at $1.5 R_{\odot}$ to $5400 \mathrm{~s}$ at $2.5 R_{\odot}$, and it was set to $3600 \mathrm{~s}$ from $2.7 R_{\odot}$ to $3.5 R_{\odot}$ ), furthermore the observations at $2.3 R_{\odot}$ and $2.5 R_{\odot}$ and at $2.7 R_{\odot}, 3.0 R_{\odot}$ and $3.5 R_{\odot}$ were summed together. For the same reason, line profiles are summed over the instantaneous field of view. Stray light correction is applied and counts are transformed to intensity, $I(\lambda)$, by applying the standard radiometric calibration (Gardner et al. 1996). The spectral line profiles, $I(\lambda)$, are fitted with a Gaussian function, representing the solar line profile, convolved with a Voigt curve which accounts for the instrumental broadening and a rectangular function accounting for the width of the spectrometer slit. The function resulting from the convolution is added to a background linearly dependent on wavelength. The best fit is obtained by applying the least square method with the following quantities as adjustable parameters: standard deviation, $\sigma$, and mean wavelength, $\lambda_{o}$, of the solar profile, and slope and intercept of the background. Finally, the observed line intensity is derived as the integral over the Gaussian line profile. The parameters defining the instrumental Voigt function and the corrections for stray light are derived by Giordano (1999).

\subsection{Data analysis}

The collisional and radiative components of the observed O VI 1032, 1037 line intensities are separated according to the procedure described in Sect. 2.4, accounting for their dependence on the plasma outflow velocity, $w$. When computing the functions $F\left(w_{n}\right)$ and $\Phi(\delta \lambda)$ for the O VI 1037 (Eq. (17)), the shift of the incident spectrum determined by coronal expansion induces the pumping of the O VI $1037.61 \AA$ by the two nearby chromospheric C II lines, at $1037.02 \AA$ and $1036.34 \AA$. This effect occurs when the exciting spectrum is red-shifted with respect to the absorption profiles by a $\delta \lambda$ sufficient to obtaining an overlapping of the coronal profiles of the O VI 1037.61 and the C II lines of the exciting spectrum (Noci et al. 1987; Dodero et al. 1998; Li et al. 1998). Partial pumping takes place even in the static case $(\boldsymbol{w}=0)$, due to the large line broadening of the absorbing profiles in the extended corona. We assume as exciting spectrum along the direction of the incident radiation, $I_{\mathrm{ex}}\left(v_{0}+\delta v, \boldsymbol{n}\right)$, the disk intensities of the $\mathrm{O}$ VI 1032 , 1037 and C II 1037, 1036 lines measured by Wilhelm et al. (1998b). The disk intensities are corrected to account for the limb brightening of the transition region OVI lines by considering a non-uniform distribution over the disk that approximates that observed with the SUMER spectrometer (Gabriel \& Vial, private communication).

The values on the plane of the sky of $T_{\mathrm{k}}$ and $T_{\mathrm{e}}$, are inferred from the measurements. The kinetic temperature $T_{\mathrm{k}}$ of interest in the case of resonant scattering is that along the direction of the incident radiation (Noci et al. 1987), which is not directly measured, since the angle between such direction and the 1.o.s. is larger than 48 degrees, at $1.5 R_{\odot}$ (minimum angle). The condition of isotropy is likely to be verified only in the inner corona, thus we assume a bi-Maxwellian velocity distribution for the oxygen ions (e.g., Kohl et al. 1998; Dodero et al. 1998). Along the 1.o.s., in the plane perpendicular to the radial direction, the kinetic temperature is given by the observed line profiles, $T_{\mathrm{k}, \text { l.o.s. }}=T_{\mathrm{k} \text {,obs }}$; whereas in the radial direction, the distribution width is assumed to vary from a lower limit, determined by the electron temperature, to a maximum value $T_{\mathrm{k} \text {,radial }}=T_{\mathrm{k} \text {,obs }}$, in the case of isotropic velocity distribution. The 1.o.s. kinetic temperatures of the O VI lines and the electron temperature are derived in Paper I. The $T_{\mathrm{k}}$ values vary from $6 \times 10^{6} \mathrm{~K}$ at $1.5 R_{\odot}$ to about $2 \times 10^{8} \mathrm{~K}$ at $3.1 R_{\odot}$. The electron temperature is extrapolated from the measurements obtained with CDS and SUMER in the inner part $\left(\leq 1.3 R_{\odot}\right)$ of the same coronal hole detected on May 21, where it never exceeds $1 \times 10^{6} \mathrm{~K}$ (David et al. 1998). Above $1.3 R_{\odot}$ it is assumed to decrease toward the heliospheric value (Paper I). 

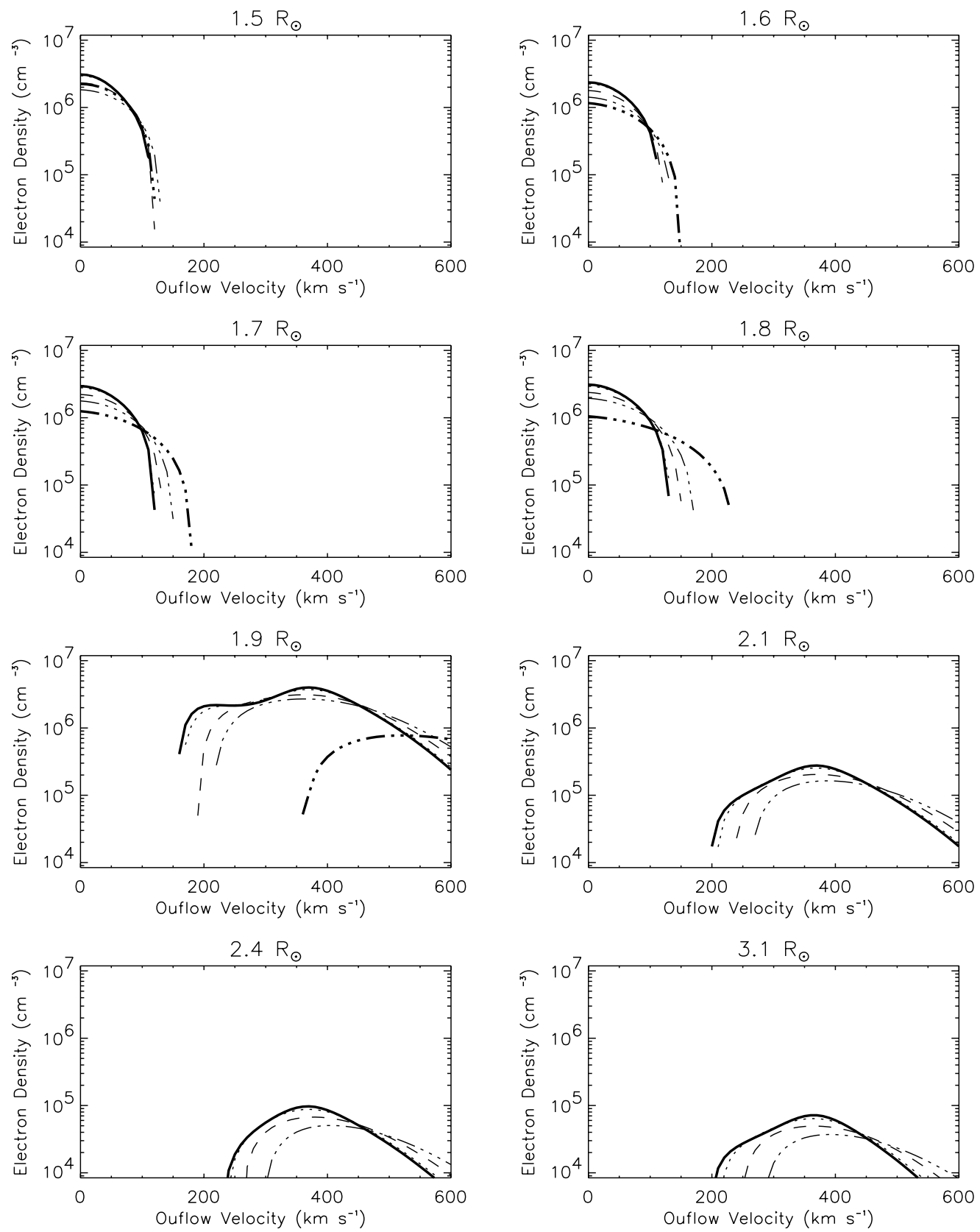

Fig. 2. Electron density of the polar coronal hole observed on May 21, 1996, as a function of outflow velocity at different heliocentric distance, derived for radial kinetic temperature $T_{\mathrm{k}, \mathrm{r}}$ equal to: $T_{\mathrm{e}}$ (bold solid lines), $T_{\mathrm{p}}$ (dotted lines), $\left(4 \times T_{\mathrm{p}}\right)$ (dashed lines), $\left(8 \times T_{\mathrm{p}}\right)($ dot-dashed lines), $T_{\text {l.os. }}$ (bold dot-dashed lines).

In the computation of the functions $F\left(w_{n}\right)$ and $\Phi(\delta \lambda)$, the degree of anisotropy in the velocity distribution of the oxygen ions adopted to describe the coronal absorbing profiles is varied in steps.

The electron density $n_{\mathrm{e}}$ is derived as a function of the outflow velocity in Eq. (13), where $I_{\mathrm{c}}(w)$ and $I_{\mathrm{r}}(w)$ are the radiative and collisional components of the O VI 1032 line. A set of $\left(n_{\mathrm{e}}, w\right)$ curves (in Fig. 2) is computed at different heights of the coronal hole. The curves differ for the degree of anisotropy of the ion velocity distribution, which is characterized by the values of radial kinetic temperature: $T_{\mathrm{k}, \mathrm{r}}$ equal to $T_{\mathrm{e}}$, bold solid lines, $T_{\mathrm{p}}$, dotted lines, $\left(4 \times T_{\mathrm{p}}\right)$, dashed lines, $\left(8 \times T_{\mathrm{p}}\right)$, dotdashed lines, and finally $T_{\mathrm{k}, \mathrm{r}}$ equal to $T_{\mathrm{obs}}$, thick dot-dashed lines. The quantity $T_{\mathrm{p}}$ is the neutral hydrogen kinetic temperature along the l.o.s., derived from the HI Lyman $\alpha$ line profiles (those values are given in Paper I), which is equal to the proton temperature in the altitude range considered in the analysis. The degree of anisotropy is progressively decreased from the maximum value here assumed, $T_{\mathrm{k}, \mathrm{r}}$ equal to $T_{\mathrm{e}}$, to reach the condition $T_{\mathrm{k}, \mathrm{r}}$ equal to $T_{\mathrm{obs}}$. At lower heights, where the oxygen ion kinetic temperatures are relatively lower, not all the defined steps need to be performed to reach isotropy.

For each $\left(n_{\mathrm{e}}, w\right)$ point, by applying the continuity equation for mass flux in a flow tube connecting the coronal hole to the heliosphere

$w(r) n_{\mathrm{e}}(r) A(r)=$ const. 

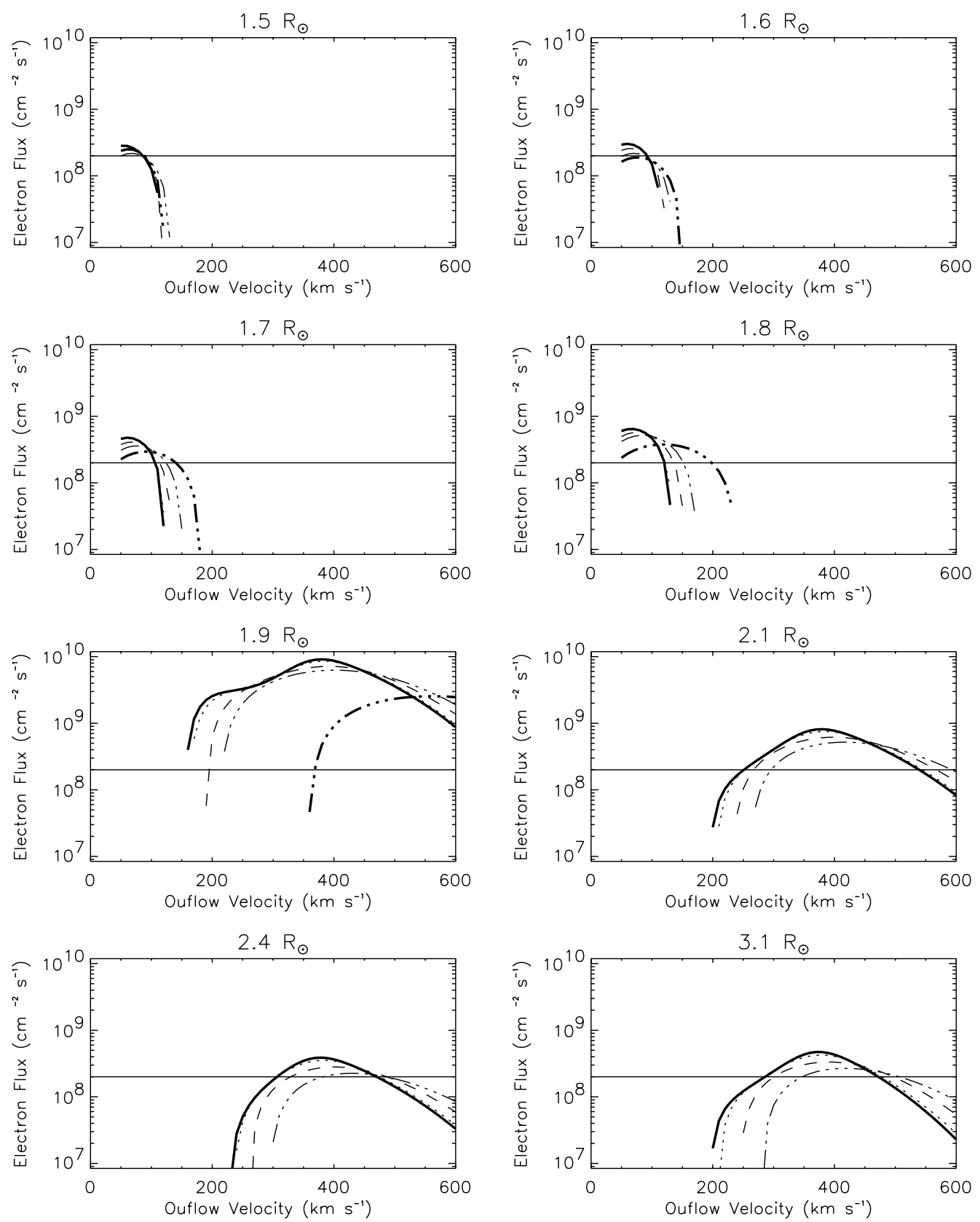

Fig. 3. Electron flux of the polar coronal hole observed on May 21, 1996, as a function of outflow velocity, extrapolated to 1 AU from the $\left(n_{\mathrm{e}}, w\right)$ curves of Fig. 2, derived for each heliocentric distance by assuming a radial kinetic temperature $T_{\mathrm{k}, \mathrm{r}}$ equal to: $T_{\mathrm{e}}$ (bold solid lines), $T_{\mathrm{p}}$ (dotted lines), $\left(4 \times T_{\mathrm{p}}\right)$ (dashed lines), $\left(8 \times T_{\mathrm{p}}\right)$ (dot-dashed lines), $T_{\text {l.o.s. }}$ (bold dot-dashed lines). The horizontal line corresponds to an electron flux $\left(n_{\mathrm{e}} \times w\right)_{1}$ AU equal to the proton flux, $2 \times 10^{8} \mathrm{~cm}^{-2} \mathrm{~s}^{-1}$, typical of the fast wind.

we derive the electron flux, $\left(n_{\mathrm{e}} \times w\right)_{1} \mathrm{AU}$ extrapolated to $1 \mathrm{AU}$ (Fig. 3). The intersections of the curves $\left[\left(n_{\mathrm{e}} \times w\right)_{1 \mathrm{AU}}, w\right]$ with the horizontal line corresponding to a value of electron flux $\left(n_{\mathrm{e}} \times w\right)_{1 \mathrm{AU}} \approx\left(n_{\mathrm{p}} \times w\right)_{1 \mathrm{AU}}=2 \times 10^{8} \mathrm{~cm}^{-2} \mathrm{~s}^{-1}$, typically observed in the fast wind streams, determine the value of the coronal density, $n_{\mathrm{e}}$, and outflow velocity, $w$, of the expanding corona in the polar coronal hole region.

In order to derive the $\left[\left(n_{\mathrm{e}} \times w\right)_{1 \mathrm{AU}}, w\right]$ curves, an appropriate flow tube geometry is defined. The white light observations are indicating that the fast wind is super-radial, with a cross-section, $A(r)$, of the flow tube that can be characterized by an expansion factor $f(r)$ greater than unity:

$A(r)=A_{0} f(r)\left(\frac{r}{r_{0}}\right)^{2}$

Hence, the flow tube geometry is assumed to depart from spherical symmetry according to the form proposed by Kopp \& Holzer (1976):

$f(r)=\frac{f_{\max } \exp \left[\left(r-r_{1}\right) / \sigma\right]+f_{1}}{\exp \left[\left(r-r_{1}\right) / \sigma\right]+1}$, 
where

$f_{1}=1-\left(f_{\max }-1\right) \exp \left[\left(r_{\mathrm{o}}-r_{1}\right) / \sigma\right]$

with $r_{\mathrm{o}}=1 R_{\odot}$, and an expansion factor with maximum value $f_{\max }=7$, close to that empirically obtained from white light observations (e.g., Munro \& Jackson 1977; Dobrzycka et al. 1999).

The parameters $r_{1}$ and $\sigma$ are varied in order to obtain a flow tube compatible with both the observed values of coronal line intensities and the heliospheric mass flux; that is, we require that the heliospheric mass flux line does intersect the $\left[\left(n_{\mathrm{e}} \times w\right)_{1 \mathrm{AU}}, w\right]$ curves (Fig. 3). Let us suppose that the superradial divergence originates in the transition region (that is, $r_{1}$ tends to $r_{o}=1 R_{\odot}$ ). In this case, only for values of $\sigma \leq 0.35$ there are physical solutions of the problem at each coronal height of the observed region (Fig. 3). If the non-radial divergence is located at higher levels (e.g. $r_{1}=1.5 R_{\odot}$ ), no value of $\sigma$ yields solutions at $1.5 R_{\odot}$ and $1.6 R_{\odot}$. That is, the curves, $\left[\left(n_{\mathrm{e}} \times w\right)_{1 \mathrm{AU}}, w\right]$, computed in this case on the basis of the observed line emission, never reach a value as high as the heliospheric electron flux one. This is an indication that the flux tubes diverge super-radially at low heights and become radial near $2.5 R_{\odot}$. In our analysis the flux tube geometry is regulated by $r_{o}=1 R_{\odot}, r_{1}=1.3 R_{\odot}, \sigma=0.3$ and $f_{\max }=7$.

\subsubsection{Electron density results}

The solutions for the plasma electron density in the acceleration region of the fast wind, inferred from the intersections of the electron flux observed at $1 \mathrm{AU}$ with that extrapolated to $1 \mathrm{AU}$ from the $\left(n_{\mathrm{e}}, w\right)$ curves, are shown in Fig. 4 . The results obtained in the case of maximum anisotropy of the oxygen ion velocity distribution $\left(T_{\mathrm{k}, \mathrm{r}}=T_{\mathrm{e}}\right.$, full dots), are approximately aligned with the density curve obtained by Guhathakurta et al. (1999). At $1.9 R_{\odot}$ the full dot corresponds to $T_{\mathrm{k}, \mathrm{r}}=T_{\mathrm{p}}$. It is to be noted that the solutions obtained for $T_{\mathrm{k}, \mathrm{r}}=T_{\mathrm{e}}$ and $T_{\mathrm{k}, \mathrm{r}}=T_{\mathrm{p}}$ are the same within the statistical errors. Therefore, since the oxygen ions decouple from protons further out in the corona than from electrons, maximum anisotropy in this context means that the oxygen ions very likely maintain along the radial direction a temperature approximately equal to the proton temperature. Whereas for an isotropic velocity distribution, solutions compatible with the observations are found only in the inner part of the extended corona, below $1.9 R_{\odot}$ (open dots). In this case, equal or lower densities than for maximum anisotropy are obtained. Above $1.9 R_{\odot}$ the assumption of isotropic velocity distribution of the oxygen ions is no longer consistent with the observations, in agreement with previous results obtained by applying Doppler dimming techniques and adopting the density independently derived from white light observations (e.g., Kohl et al. 1998; Antonucci et al. 2000). The results obtained at higher altitudes for the lowest degree of anisotropy allowed in the analysis $\left(T_{\mathrm{k}, \mathrm{r}}=8 \times T_{\mathrm{p}}\right)$ are denoted by open dots. Above $1.9 R_{\odot}$ each curve $\left[\left(n_{\mathrm{e}} \times w\right)_{1 \mathrm{AU}}, w\right]$ intersects twice the horizontal line corresponding to the electron flux of the fast wind observed at $1 \mathrm{AU}$, in the velocity range from $0 \mathrm{~km} \mathrm{~s}^{-1}$ to $600 \mathrm{~km} \mathrm{~s}^{-1}$ (Fig. 3). The density solutions relative to the

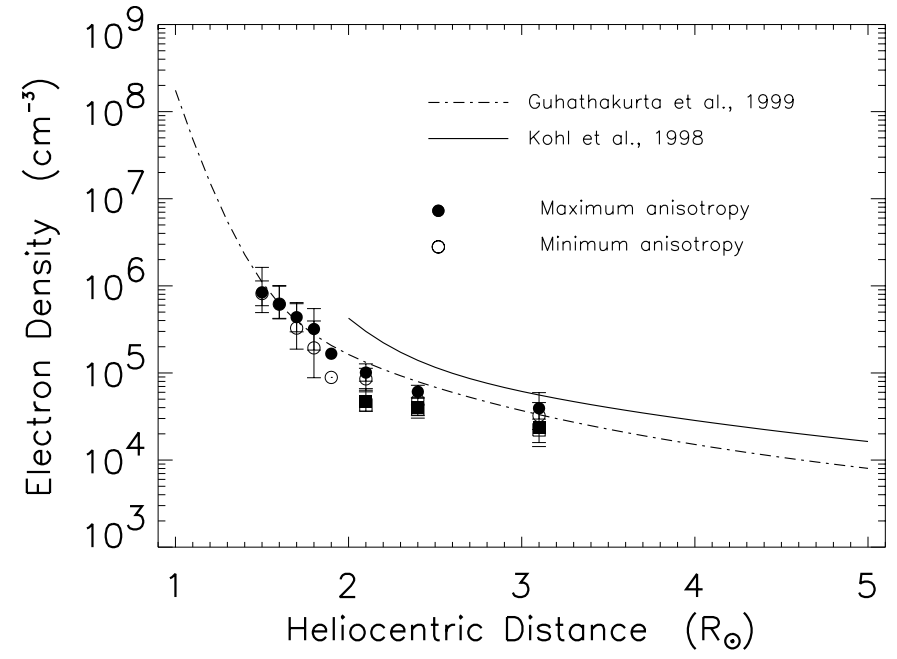

Fig. 4. Electron density of the polar coronal hole observed on May 21, 1996, as a function of height in the extended corona, derived with the spectroscopic technique based on the OVI line intensities. The present results are compared with density results obtained from white light observations. Full dots correspond to the case of maximum anisotropy of the oxygen ion velocity distribution. Only at $1.9 R_{\odot}$ the full dot corresponds to $T_{\mathrm{k}, \mathrm{r}}=T_{\mathrm{p}}$. Open dots correspond to isotropic ion velocity distribution out to $1.9 R_{\odot}$, and beyond this height to the lowest degree of anisotropy allowed in the analysis, $T_{\mathrm{k}, \mathrm{r}}=8 \times T_{\mathrm{p}}$. Full and open squares, mostly overlapping, indicate the results derived from the second intersection at higher velocity (in Fig. 3), for maximum and minimum anisotropy, respectively.

second intersection, corresponding to higher velocity, are indicated as squares in Fig. 4. Full and open squares denote maximum and minimum anisotropy, respectively, as in the case of the results obtained from the first intersection (Fig. 3). The error bars of the density values result from the propagation of the statistical uncertainties of the observed OVI 1032, 1037 line intensities. The density results however also depend on other factors, those effects are addressed in what follows.

The disk intensity of the exciting spectrum, affecting the density through the quantity $F(w)$, is seldom measured and in general not at the time of the coronal observations. However when computing the coronal densities by using the OVI disk intensity values obtained during solar minimum by Raymond et al. (1997), which exceed by a factor of 1.5 and 1.2 those observed by Wilhelm et al. (1998b) and Vernazza \& Reeves (1978), respectively, the variation of the density results is relatively small $(\approx 10 \%)$.

In the altitude range from 1.5 to $3.1 R_{\odot}$, the rapidly expanding plasma can depart from ionization equilibrium, since the expansion time in this region is shorter than the oxygen ionization time. However, this does not affect the present results since the density is proportional to the ratio of the collisional and radiative component of an oxygen line, which in turn are both proportional to the oxygen ion column density and therefore to the oxygen ionization fraction.

The uncertainty in deriving the plasma density on the plane of the sky from the OVI doublet intensities has been already addressed in Sect. 2.3. 
Hence the largest effect on the density results is indeed deriving from the limited direct information on the velocity distribution along the incident radiation of the oxygen ions, which resonantly scatter the ultraviolet disk emission in the extended corona.

\subsubsection{Dependence on the filling factor}

Let us also consider the influence on the results of the presence of an inhomogeneous plasma in the region of acceleration of the fast wind, with high density $\left(n_{\mathrm{e}, \text { thread }}, n_{i, \text { thread }}\right)$ threads embedded in a thinner ambient atmosphere $\left(n_{\mathrm{e}}^{\prime}, n_{i}^{\prime}\right)$. We define a filling factor, $f=V_{\text {thread }} / V$, representing the fraction of unresolved volume occupied by the denser elements and a contrast ratio $c=n^{\prime} / n_{\text {thread }}$. Noci et al. (1997) explicitly gives the dependence of the radiative and collisional components on the filling factor, $f$. The ratios $R_{1}$ and $R_{2}$ do not depend on the filling factor, hence the separation between collisional and radiative component of the observed lines is not affected by the presence of inhomogeneities in the coronal plasma. On the other hand since the electron density is proportional to the ratio $\frac{I_{c}}{I_{\mathrm{r}}}$ (Eq. (13)), for an inhomogeneous plasma we obtain

$n_{\mathrm{e}, \text { thread }}=\frac{[f+c(1-f)]}{\left[f+c^{2}(1-f)\right]} n_{\mathrm{e}}$,

where $n_{\mathrm{e}}$ is the quantity derived via the present spectroscopic technique (Eq. (13)). In the assumption of a filling factor $f=$ 0.1 and a ratio $c=0.1$, the electron density of the thicker threads is almost twice that derived with the OVI spectroscopic method (Fig. 4). On the other hand the thread density would result approximately 5 times larger than the average plasma density defined as:

$\bar{n}_{\mathrm{e}}=\frac{n_{\mathrm{e}, \text { thread }} V_{\text {thread }}+n^{\prime} V^{\prime}}{V_{\text {thread }}+V^{\prime}}$,

where $V=V_{\text {thread }}+V^{\prime}$ is the total volume. Hence

$n_{\mathrm{e}, \text { thread }}=\frac{1}{[f+c(1-f)]} \bar{n}_{\mathrm{e}}$.

Therefore while for a homogeneous solar wind the electron density derived spectroscopically is equal to the average density, $n_{\mathrm{e}}=\bar{n}_{\mathrm{e}}$, in the case that the wind plasma is formed by higher density elements embedded in a thinner ambient atmosphere flowing at the same speed, the density derived spectroscopically from the OVI lines is larger than the average density by a factor that depends on the filling factor, $f$, and the concentration ratio, $c=n^{\prime} / n_{\text {thread }}$

$n_{\mathrm{e}}=\frac{\left[f+c^{2}(1-f)\right]}{[f+c(1-f)]^{2}} \bar{n}_{\mathrm{e}}$.

For $f=0.1$ and $c=0.1$ the density derived spectroscopically is larger than the average density by a factor $\sim 3$. Hence, if the solar wind is filamentary and the outflow velocity is considered mainly homogeneous, the density values obtained in Fig. 4 overestimate the average wind density; that is, average coronal density values lower than in Fig. 4 should be expected.

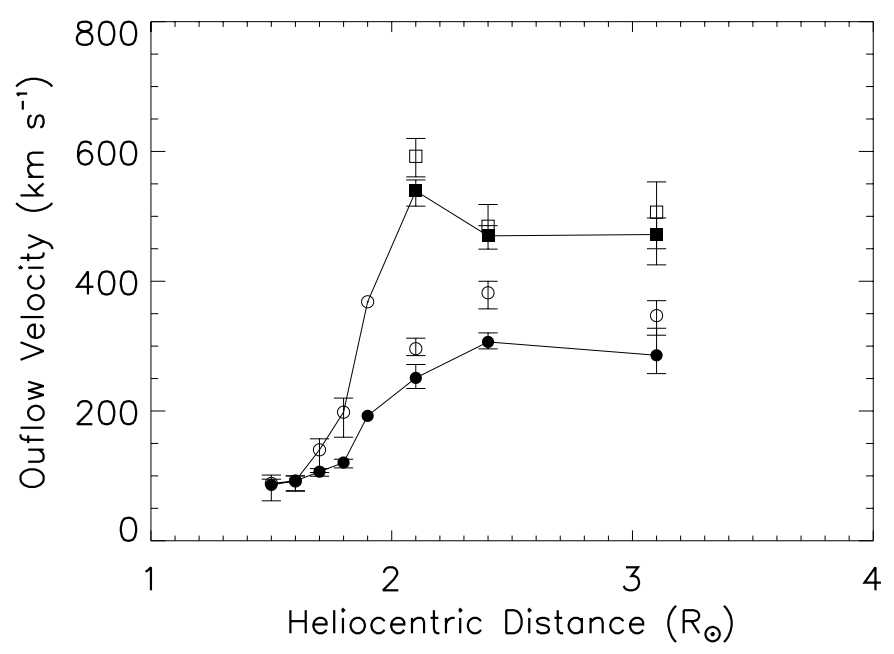

Fig. 5. Outflow velocity of the polar coronal hole observed on May 21, 1996, as a function of height in the extended corona, derived with the spectroscopic technique based on the OVI line intensities. Full dots correspond to the case of maximum anisotropy of the oxygen ion velocity distribution. Only at $1.9 R_{\odot}$ the full dot corresponds to $T_{\mathrm{k}, \mathrm{r}}=T_{\mathrm{p}}$. Open dots correspond to isotropic ion velocity distribution out to $1.9 R_{\odot}$, and beyond this height to the lowest degree of anisotropy allowed in the analysis, $T_{\mathrm{k}, \mathrm{r}}=8 \times T_{\mathrm{p}}$. Full and open squares, mostly overlapping, indicate the results derived from the second intersection at higher velocity (in Fig. 3), for maximum and minimum anisotropy, respectively.

\subsubsection{Outflow velocity results}

The analysis of the outflow velocity results, reported in Fig. 5, shows that in the inner part of the extended corona, around $1.5-1.6 R_{\odot}$ the outflow velocity is close to $100 \mathrm{~km} \mathrm{~s}^{-1}$, independently of the velocity distribution assumed for the oxygen ions along the radial direction. Beyond $1.7 R_{\odot}$ the expansion velocity is derived with a relatively large uncertainty that depends on the radial kinetic temperature of the ions. If the velocity distribution is assumed to be isotropic (open dots out to $1.9 R_{\odot}$ ), the rate of expansion of the corona is higher than for ion radial kinetic temperatures close to that of electrons indicated by full dots (or to protons, the outflow velocity obtained in the two cases being the same within the statistical uncertainty).

Further out, where the observed kinetic temperature along the l.o.s. is progressively increasing to very large values, of the order of $10^{8} \mathrm{~K}$, no solutions are found for a fully isotropic ion distribution (above $1.9 R_{\odot}$ open dots correspond to a radial kinetic temperature $T_{\mathrm{k}, \mathrm{r}}=8 \times T_{\mathrm{p}}$ ). Beyond $1.9 R_{\odot}$, there exists also a higher velocity solution obtained from the second intersection in Fig. 3. The high-velocity solutions, when considering the statistical uncertainty, are less dependent on the assumptions on the radial kinetic temperature. In Fig. 5, full squares correspond to maximum anisotropy, with radial kinetic temperature equal to electron temperature; open squares correspond to minimum anisotropy with $T_{\mathrm{k}, \mathrm{r}}=8 \times T_{\mathrm{p}}$. The area delimited by the two curves (full lines), joining the point obtained in the limiting cases of ion velocity distribution, corresponds to acceptable solutions obtained for intermediate bi-Maxwellian distributions. 
In the assumption that in the extended corona the ions maintain a positive acceleration, there exist more than one acceptable outflow velocity curve for the fast solar wind. A lower curve can be drawn by joining the values obtained for the highly anisotropic ion velocity distribution $\left(T_{\mathrm{k}, \mathrm{r}}=T_{\mathrm{e}}\right.$, full dots) up to approximately $2.4 R_{\odot}$, and, further out, the value obtained when the velocity distribution relaxes to a lesser degree of anisotropy (open dots above $2.4 R_{\odot}$ ). Along this curve the ions increase their radial temperature, from a value close to the electron/proton temperature, only above $2.4 R_{\odot}$, and the velocity reached at $3.1 R_{\odot}$ is approximately $350 \mathrm{~km} \mathrm{~s}^{-1}$.

Alternatively we can draw a curve that from the values obtained for high anisotropy out to $2.1 R_{\odot}$, reaches the values obtained in the same hypothesis, when considering the second intersection in Fig. 3, at 3.1 $R_{\odot}$, where the outflow velocity approaches $500 \mathrm{~km} \mathrm{~s}^{-1}$. In this second case, there is no increase of the radial temperature of the oxygen ions out to $3.1 R_{\odot}$ and the acceleration is higher.

The first two curves of growth of the outflow velocity of the fast solar wind imply therefore a high anisotropy ratio, $T_{\text {l.o.s. }} / T_{\mathrm{r}}$, for the velocity distribution of the oxygen ions. The ratio is increasing throughout the region of observation from 1.5 to $3.1 R_{\odot}$, to values of the order of 100 , in the second of the two cases. It increases in a more limited region out to $2.4 R_{\odot}$, in the first case, where the anisotropy ratio reaches a maximum ( $\sim 100)$ and then decreases. (It has to be noted however that the high velocity points, $\sim 500 \mathrm{~km} \mathrm{~s}^{-1}$, are not very sensitive to the anisotropy ratio, therefore it is also possible that at $3.1 R_{\odot}$ an isotropy condition is reached.) The anisotropy ratio is expressed in terms of kinetic temperatures relative to the direction of the coronal magnetic field, which lies approximately along the radial direction in the polar regions during solar minimum (that is, $T_{\text {l.o.s. }} / T_{\mathrm{r}} \sim T_{\perp} / T_{\|}$relative to the coronal field lines).

A completely different physical situation, however, is also compatible with the observations and the requirement of positive acceleration of the solar wind, that can be represented by a third curve joining the points obtained for isotropic ion velocity distribution out to 1.8 solar radii and those obtained for the lowest degree of anisotropy above $1.9 R_{\odot}$ (open dots). In this case the anisotropy ratio diverges from unity above $1.8 R_{\odot}$ and increases to a value of the order of 10 above $1.9 R_{\odot}$. The third curve, in order to maintain positive acceleration should reach the high velocity point at $3.1 R_{\odot}$.

The three curves are representative of the limiting conditions compatible with the data and the constraint of positive acceleration; intermediate conditions of course are also possible. On the other hand, the points within the area above the three most representative curves discussed above are likely to be excluded if the oxygen component of the solar wind is positively accelerated out to 3 solar radii.

The outflow velocities (Fig. 5) derived with the spectroscopic method proposed in this paper are consistent with the results obtained in the Doppler dimming analysis performed in Paper I, when using the lower density corona values derived by Guhathakurta et al. (1999).

The velocity results of Fig. 5 depend on the assumption of a homogeneous fast solar wind. The presence of any unresolved filamentary structure would lead to overestimate the density. Thus for a filamentary solar wind the density averaged over thin and thick unresolved elements would be lower and, as a consequence, the outflow velocity would result to be higher than in Fig. 5.

\section{Conclusions}

The spectroscopic diagnostics of the extended corona developed in this paper based on the collisional and radiative components of the OVI 1032 and 1037 lines, when applied to the coronal regions where the solar wind accelerates, allows us to derive both the electron density and the outflow velocity, given the constraint of mass flux conservation in the flow tube connecting the open field corona to the heliosphere. When applied to a static coronal plasma, this method allows to account for the effect of the pumping of the OVI 1037 line by the two nearby CII lines, that in this case occurs due to the broad coronal absorption profiles existing in the extended corona.

The results obtained with this diagnostic technique are influenced by the assumptions on the geometry of the flux tube; thus they can also give information on the divergence of the field lines. In order to find solutions for electron density and outflow velocity in the entire range from $1.5 R_{\odot}$ to $3.1 R_{\odot}$, given the OVI 1032-1037 line intensities observed in polar coronal hole during solar minimum, the super-radial divergence of the magnetic field lines has to initiate in the transition region/inner corona. In addition the flow tube diverges rapidly, reaching radial expansion close to $2.5 R_{\odot}$. Hence this result is consistent with the idea of open magnetic field lines originating close to the network boundaries and spreading out in the transition region to fill almost homogeneously the corona, according to the model suggested by Gabriel (1976).

The electron density obtained with this method is in agreement with the visible light density measurements performed in coronal holes by Ghuathakurta et al. (1999), thus indicating the existence in coronal holes of a thinner plasma than derived from other visible light measurements. The presence along the 1.o.s. of density concentrations in an ambient thin plasma would induce an overestimate of the average electron density.

The electron density and outflow velocity results obtained from the resonantly scattered ultraviolet emission observed in the extended corona, depend on the coronal absorption profiles along the direction of the incident radiation, which is not directly measurable. The dependence however is much larger for what concerns the velocity results. The velocity values obtained here are consistent with those derived for a thin corona in Paper I, and therefore with the conclusions pertaining to this case. That is, the outflow velocity of the fast solar wind in polar holes can be traced by the motion of the O VI ions at least up to $2.4 R_{\odot}$, since the Doppler dimming analysis of the $\mathrm{O} \mathrm{VI}$ and $\mathrm{H}$ I lines in a low density corona, yields a flow speed of oxygen ions and protons along the open magnetic field lines with roughly the same value. Beyond this height, depending on the assumptions on the coronal absorbing profiles of the scattering ions along the direction of the incident radiation the oxygen flow velocity might tend to be higher than the neutral hydrogen outflow velocity. 
According to the discussion of Sect. 3.2.3, a plausible interpretation of the results on the outflow velocity of the coronal plasma in polar holes implies high anisotropy of the ion velocity distribution. The oxygen ions maintain, throughout the observed altitude range, kinetic temperatures along the coronal magnetic field close to proton temperatures, being the field roughly aligned with the radial direction in polar regions. As the oxygen kinetic temperature across the magnetic field increases with height to values above $1 \times 10^{8} \mathrm{~K}$, the degree of anisotropy in the ion velocity distribution increases between $1.5 R_{\odot}$ and $3.1 R_{\odot}$ to very high values $\left(T_{\perp} / T_{\|} \approx 100\right)$. As a consequence the heating process active in this layer of the solar corona accelerates the ions across the magnetic field, at a degree increasing with height. Furthermore the released energy remains mainly in the direction across the magnetic field without significant transfer to the radial direction. A variant of this interpretation allows, beyond $2.4 R_{\odot}$ the solution relative to a lower degree of anisotropy of the ion velocity distribution; then at these heights a gradual transfer to the radial direction of the energy deposited across the magnetic field is observed (the anisotropy ratio decreases to 10-20). An alternative interpretation of the velocity of the oxygen component of the fast wind is also possible, which implies instead a condition of isotropy of the ion velocity distribution out to $1.8 R_{\odot}$, beyond this point a moderate anisotropy has to be invoked, with an anisotropy ratio, however, not exceeding 20 . The different interpretations depict quite different scenarios. On the one hand, the process accelerating the ions across the magnetic field originates in the inner corona, is extremely efficient from 1.5 to $3.1 R_{\odot}$ and the energy remains substantially confined in the direction perpendicular to the field. On the other limiting case, the process accelerating the ions preferentially across the magnetic field originates further out in the extended corona beyond $1.8 R_{\odot}$, and it is efficient beyond $2.1 R_{\odot}$. In both cases high outflow velocities of the oxygen component of the fast wind can be reached within $3.1 R_{\odot}\left(500 \mathrm{~km} \mathrm{~s}^{-1}\right)$, but the acceleration is larger between 1.7 and $2.4 R_{\odot}$ when isotropic conditions are maintained for an extended altitude range.
Acknowledgements. UVCS is a joint project of the National Aeronautics and Space Administration (NASA), the Agenzia Spaziale Italiana (ASI) and Swiss Founding Agencies. This work was supported by ASI grants (I/R/125/01 and $\mathrm{I} / \mathrm{R} / 084 / 02)$ and the contract MIUR-COFIN-2001.

\section{References}

Antonucci, E., Dodero, M. A., \& Giordano, S. 2000, Sol. Phys., 197, 115

David, C., Gabriel, A. H., Bely-Dubau, F., et al. 1998, A\&A, 336, L90 Dobrzycka, D., Cranmer, S. R., Panasyuk, A. V., Strachan, L., \& Kohl, J. L. 1999, JGR, 104(A5), 9791

Dodero, M. A., Antonucci, E., Giordano, S., \& Martin, R. 1998, Sol. Phys., 183, 77

Elwert, G. 1952, Z. Naturforsch., 7a, 432

Fisher, R., \& Guhathakurta, M. 1995, ApJ, 447, L139

Gabriel, A. H. 1976, Philos. Trans. R. Soc. London Ser. A, 281, 339

Gabriel, A. H., \& Jordan, C. 1972, Case Studies in Atomic Collision Physics, Vol. 2, ed. McDaniel \& McDowell (North Holland)

Gardner, L. D., Kohl, J. L, Daigneau, P. S., et al. 1996, Proc. SPIE, 2831, 2

Giordano, S. 1999, Ph.D. Thesis, Università degli Studi di Torino

Guhathakurta, M., Fludra, A., Gibson, S. E., Biesecker, D., \& Fisher, R. 1999, JGR, 104(A5), 9801

Kopp, R. A., \& Holzer, T. E. 1976, Sol. Phys., 49, 43

Kohl, J. L., Noci, G., Antonucci, E., et al. 1998, ApJ, 501, L127

Koutchmy, S. 1977, Sol. Phys., 51, 399

Li, X., Habbal, S. R., Kohl, J. L., \& Noci, G. 1998, ApJ, 501, L133

Munro, R. H., \& Jackson, B. V. 1977, ApJ, 213, 874

Noci, G., Kohl, J. L., \& Withbroe, G. L. 1987, ApJ, 315, 706

Noci, G., Kohl, J. L., Antonucci, E., et al. 1997b, Fifth SOHO workshop, ESA SP-404, 75

Raymond, J. C., Kohl, J. L., Noci, et al. 1997, Sol. Phys., 175, 645

Vernazza, J. E., \& Reeves, E. M. 1978, ApJ, 37, 485

Wilhelm, K., Marsch, E., Dwivedi, B. N., et al. 1998a, ApJ, 500, 1023

Wilhelm, K., Lemaire, P., Dammasch, I. E., et al. 1998b, A\&A, 334, 685 